\title{
A DATA-DRIVEN APPROACH TO USER-EXPERIENCE- FOCUSED MODEL-BASED ROADMAPPING FOR NEW PRODUCT PLANNING
}

\author{
Iuskevich, Ilia (1,2); \\ Hein, Andreas-Makoto (2); \\ Amokrane-Ferka, Kahina (2); \\ Doufene, Abdelkrim (2); \\ Jankovic, Marija (1) \\ 1: Université Paris-Saclay, CentraleSupélec, Laboratoire Genie Industriel; \\ 2: IRT SystemX
}

\begin{abstract}
User experience (UX) focused business needs to survive and plan its new product development (NPD) activities in a highly turbulent environment. The latter is a function of volatile UX and technology trends, competition, unpredictable events, and user needs uncertainty. To address this problem, the concept of design roadmapping has been proposed in the literature. It was argued that tools built on the idea of design roadmapping have to be very flexible and data-driven (i.e., be able to receive feedback from users in an iterative manner). At the same time, a model-based approach to roadmapping has emerged, promising to achieve such flexibility. In this work, we propose to incorporate design roadmapping to model-based roadmapping and integrate it with various user testing approaches into a single tool to support a flexible data-driven NPD planning process.
\end{abstract}

Keywords: Organisation of product development, Product modelling / models, New product development, Technology Roadmapping, Strategic management

\section{Contact:}

Iuskevich, Ilia

Université Paris-Saclay

Le Laboratoire Génie Industriel CentraleSupélec

France

ilya.yuskevich@gmail.com

Cite this article: Iuskevich, I., Hein, A.-M., Amokrane-Ferka, K., Doufene, A., Jankovic, M. (2021) 'A Data-Driven Approach to User-Experience-Focused Model-Based Roadmapping for New Product Planning', in Proceedings of the International Conference on Engineering Design (ICED21), Gothenburg, Sweden, 16-20 August 2021. DOI:10.1017/ pds.2021.7 


\section{INTRODUCTION}

Technology roadmapping (TRM) is one of the most popular methods in the field of strategic management. It is visual and easy to understand. Within TRM it is possible to aggregate large amounts of data and capture many facets of company activities. Since this method was proposed, it was considered mainly as a tool for long-term planning in large corporations and agencies.

A typical example of TRM is the 2015 NASA Technology Roadmap (Archive) (2015). It deals with incredibly complex, risky, and expensive projects, which, however, are executed in a financially stable environment with low competition. Under such conditions, planning is possible for years and even decades ahead.

User-oriented startups and their more established rivals reside in a different environment. Even though their products usually have a relatively simple architecture, the business environment around them bears many unknowns. It is turbulent, uncertain, and highly competitive. Moreover, the final requirements for the products are often ambiguous and evolving.

A number of approaches were proposed in the field of innovation management to improve the chances of a user experience (UX) focused business to succeed. The most prominent are lean startup (Ries, 2011) and design thinking (Buchanan, 1992; Meinel et al., 2020). At their core, these approaches rely on a constant adaptation of products and business models through iterative prototyping and experimentation.

Kim et al. (2016) proposed to adapt TRM to the reality of the UX-focused business under the label of design roadmapping. Not denying, but complementing agile approaches, design roadmapping emphasizes the importance of prototyping and experimentation and adds short- and mid-term planning elements. It promotes the idea of "roadmapping for future preparation, not prediction" (Kim et al., 2018). The roadmap must be maintained as a flexible and interactive, 'living' document, which is constantly modified as new information becomes available after, for example, a new round of user testing.

Model-based technology roadmapping (MB-TRM) is another recently proposed approach (Golkar and Garzaniti, 2020; Gradini et al., 2019; Knoll et al., 2018), which promises to meet the needs of design roadmapping. It relies on a general paradigm shift in engineering, which is a substitution of documents with interactive computer models. The formal syntax underlying these models enables several features required for design roadmapping, i.e., interactivity, modifiability, flexibility, concurrency when used by several people. An ontology of concepts (semantics) provides common definitions to all involved stakeholders. The biggest challenge is designing this formal syntax and defining semantics to represent the problem adequately.

Thereby, this paper aims to propose one possible solution that will represent the complex and often ambiguous nature of UX-focused business and help in its NPD planning. To achieve this, we will need to capture and model UX-related concepts such as personas, use cases, user goals, and more technical concepts such as product family architectures. A specific set of NPD processes and concepts integrated into our MB-TRM framework was selected to be easily deployed in the automotive industry context. We demonstrate the approach on the example of a semi-automated vehicle cockpit design.

\section{LITERATURE REVIEW}

Designing a roadmapping solution for managing UX-focused business is a multifaceted task due to the roadmaps' fundamentally cross-domain nature. In the following, we will review the TRM literature, as our main focus is roadmapping. In addition, we cover literature proposing product models, product family models, and user testing, as these elements are necessary for integrating product development and UX concepts into roadmapping.

Several papers already presented approaches aiming to integrate TRM with new product development tools. Phaal et al. (2005) argue that TRM is a good candidate for combining other strategic tools and processes as "common reference point for ongoing discussion, and a place to store information." In their paper, they demonstrated the integration of TRM with the linked grid approach. An et al. (2008) incorporated Quality Function Deployment to TRM for product-service development. Bekhradi et al. (2018) developed a TRM-based methodology to help technology-based startups allocate their resources to $R \& D$ activities.

Iuskevich et al. (2021) proposed a metamodel for a model-based roadmap. In this metamodel, elements of a roadmap exist in a three-dimensional space (time-level-scenario) and are subject to classification (e.g., products, markets, personas, use cases, etc.). Each element may be associated with 
several quantitative attributes and models. Models are the most important concepts for this central integrating role of MB-TRM. They provide an interface for other NPD processes - user tests, product models, business models, personas, etc.

Products and (or) services are central elements of design roadmapping. Several product models have been proposed in the literature. QFD represents products and services through the House of Quality and a roof matrix (An et al., 2008; Franceschini and Rossetto, 1995; Shen et al., 2000). It is also common in the systems engineering field to represent different product facets by separate models. For instance, Helms and Shea (2012) propose to define Function-Behavior-Structure of a product with object-oriented graph grammars. Wu et al. (2013) applied SysML diagrams to a product variety management problem.

$\mathrm{Du}$ et al. (2001) proposed an approach to product family architecting. An entire product family is modeled with a special visual language called Generic Product Structure (GPL), whereas individual products are configured as instances of a product family model.

A Feature-oriented Domain Analysis (FODA) is an approach equivalent to the described above. It appeared as a tool for software product lines architecting decades ago, and today, it is thoroughly developed (Nešić et al., 2019). It utilizes the same idea of modeling individual products through a product family and has a well-defined syntax for representing mandatory, optional, alternative features, and cross-tree constraints. Many extensions of FODA were proposed, both in terms of syntax and application domains. Above all, we are interested in the application of FODA to the new product development domain. Abrantes and Figueiredo (2014) employed FODA to managing NPD portfolios in the semiconductor industry. Zhou et al. (2017) used FODA to represent customer needs and functional requirements of a consumer electronics device and integrated it with the sentiment analysis for the product assessment.

Another essential component of our proposed framework is user testing. Within this paper scope, we will discuss three out of many possible options, namely conjoint analysis (CA), Kansei engineering, and our ad-hoc version of the point allocation method applied to user stories.

$\mathrm{CA}$ is a standard tool in marketing to determine customer preferences toward different product versions. This approach was practiced and studied for over half a century (Green et al., 2001). The abundance of data on CA makes it extremely trustworthy and appealing to apply. Nowadays, researchers from design science and systems engineering actively integrating CA into their frameworks. Colombo et al. (2020) proposed to use conjoint analysis to determine the user value of customizable modular products. Kim et al. (2017) broadened the use of conjoint analysis to experimentation with potential user experiences on early-stage product development. Muller and Lillack (2011) demonstrated seamless compatibility of CA and FODA. The latter is directly incorporated into our approach.

$\mathrm{CA}$ is used to estimate the perceived value of concrete features included in a product version. Nevertheless, a single resulting score provided by CA does not offer sufficient insights into the user reasoning. The majority of the UX conceptual models emphasize the importance of the distinction between the emotional and pragmatic components of the UX (e.g., pragmatic and hedonic qualities in the model proposed by Hassenzahl (2018)). A product's aesthetics is often described using adjectives with opposite meanings (e.g., progressive-conservative, formal-casual, etc.), which is the basic idea behind Kansei engineering (KE) (Nagamachi, 2002). We use KE to cluster users by their emotional profiles and perform personalized assessments of a product's aesthetics as described in Kett et al. (2017).

Finally, the notion of UX is not limited to the interaction between a user and a product. The context in which this interaction happens greatly influences a user's judgment on product qualities. For this reason, we incorporated the concept of user stories. As defined in Alexander and Maiden (2004), a user story refers to a "Scenario format used in some Agile Methods consisting of a brief and often informal narrative text (a Story) that typically mentions an Actor and the actor's Goal, with a description, possibly by example, of how the goal can be achieved." In our approach, participants assess different user stories with the point allocation method. The advantages and downturns of the latter are discussed in Doyle et al. (1997).

Our literature review shows that prior research efforts aimed to develop diverse NPD methodologies and establish pairwise integration between them. It seems promising to us to propose a modular framework that will integrate diverse NPD approaches for a given context. We argue that, first, MBTRM is a good candidate to become a platform for combining different modules. More specifically, our approach provides a mapping between UX-related concepts (personas, goals, use cases), productrelated concepts (features), and user testing procedures (CA, KE, point allocation), which naturally 
ease roadmapping-related procedures (features prioritization, product line planning, envisioning, etc.). Ultimately, our approach promises to provide an instrument for storing all NPD information in one place and facilitating an iterative routine consisting of design (in terms of product and UX modeling), user testing, and planning.

\section{A UX-FOCUSED MODEL-BASED ROADMAPPING}

We integrate UX concepts into the MB-TRM metamodel from Iuskevich et al. (2021) and subsequently add sample NPD processes to the resulting UX-focused design roadmap in order to illustrate the application of our approach.

The MB-TRM metamodel developed by Iuskevich et al. (2021) is shown in Figure 1. This metamodel can represent data models of a large variety of MB-TRM applications.

Element is a key concept and building block of any roadmap. Elements are located on the different Levels of a roadmap, have a start and end date, and may belong to different scenarios (Pessimistic or Optimistic). For our context, we defined seven classes of elements (ElementClass) - UX Trend, Persona, Use case, User Goals, UX Test, Product, and Technology.

Some ElementClasses of the roadmap have a model. For the modeling, we chose to use FODA. A feature tree represents an entire product family, a family of user goals, use cases, and personas' traits. A feature tree configuration (ModelConfiguration) represents a specific product, persona, set of user goals, and use cases.

This minimal example illustrates how the MB-TRM metamodel enriched with FODA syntax and specified by UX-related concepts results in a meaningful framework aiming to facilitate collaboration between stakeholders, elicitation of user opinions, and NPD planning. This framework can be extended by request, as shown in figure 2 (marked modules are implemented in our software prototype). By adding new ElementClasses and Levels and associating them with different Models, the framework may be adapted to a particular context in order to aggregate, store and process additional information on products, user profiles, business models, technologies, etc.

Figure 3 illustrates the idea of UX-related concepts modeling with feature trees (the scope of these trees is simplified compared to the real project).

\section{SAMPLE APPLICATION}

Based on this methodology, a web-based software tool was developed and deployed in the cloud. We illustrate this tool functionality with the design of a semi-automated vehicle human-machine interface. An exemplary roadmap for this use case is shown in Figure 4.

Note: in order to protect sensitive commercial information from disclosure, we altered feature names, some user goals, and use cases. We also shuffled the sequence of the aggregated user responses with respect to the assessed features while their numerical values remain the same.

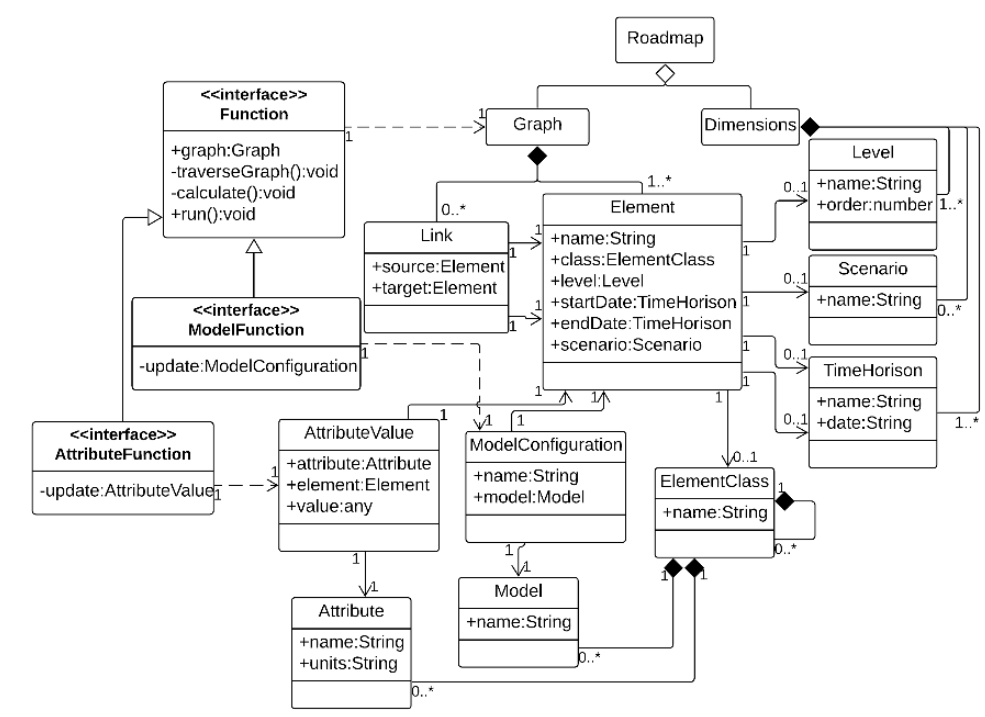

Figure 1. MB-TRM metamodel (from Luskevich et al. (2021)) 


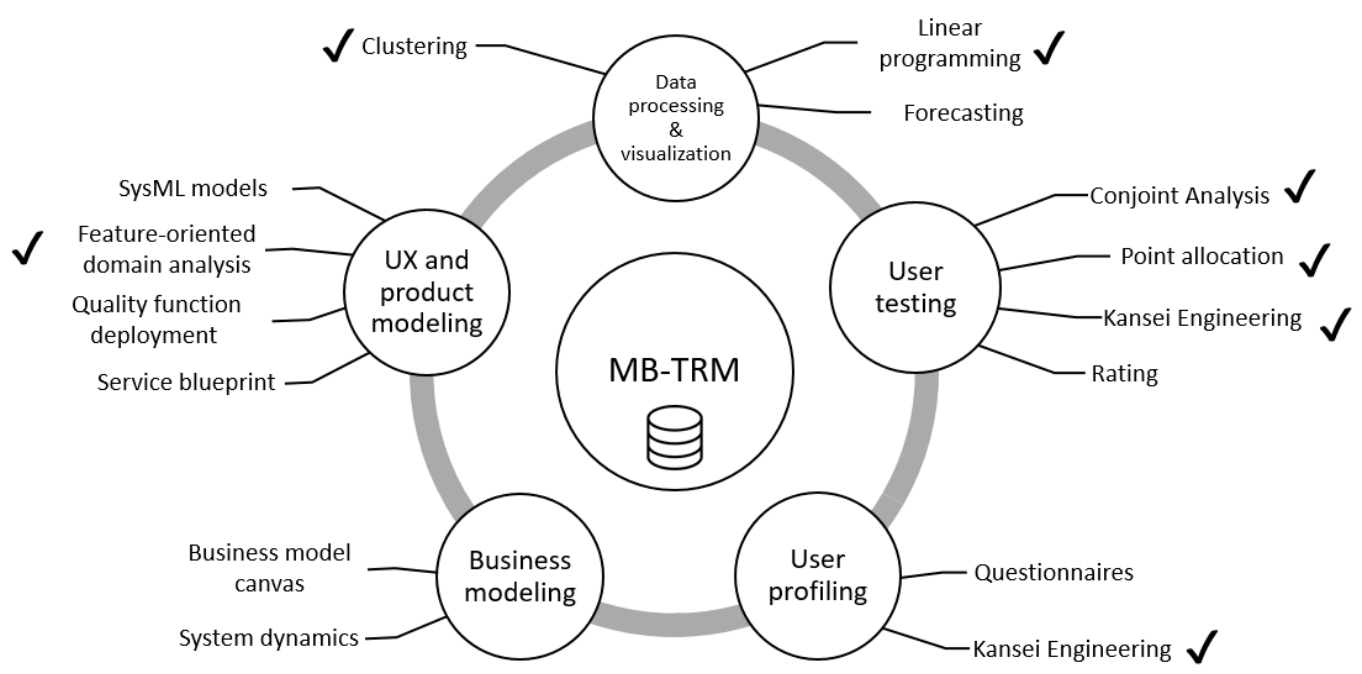

Figure 2. MB-TRM as a platform for integration NPD processes
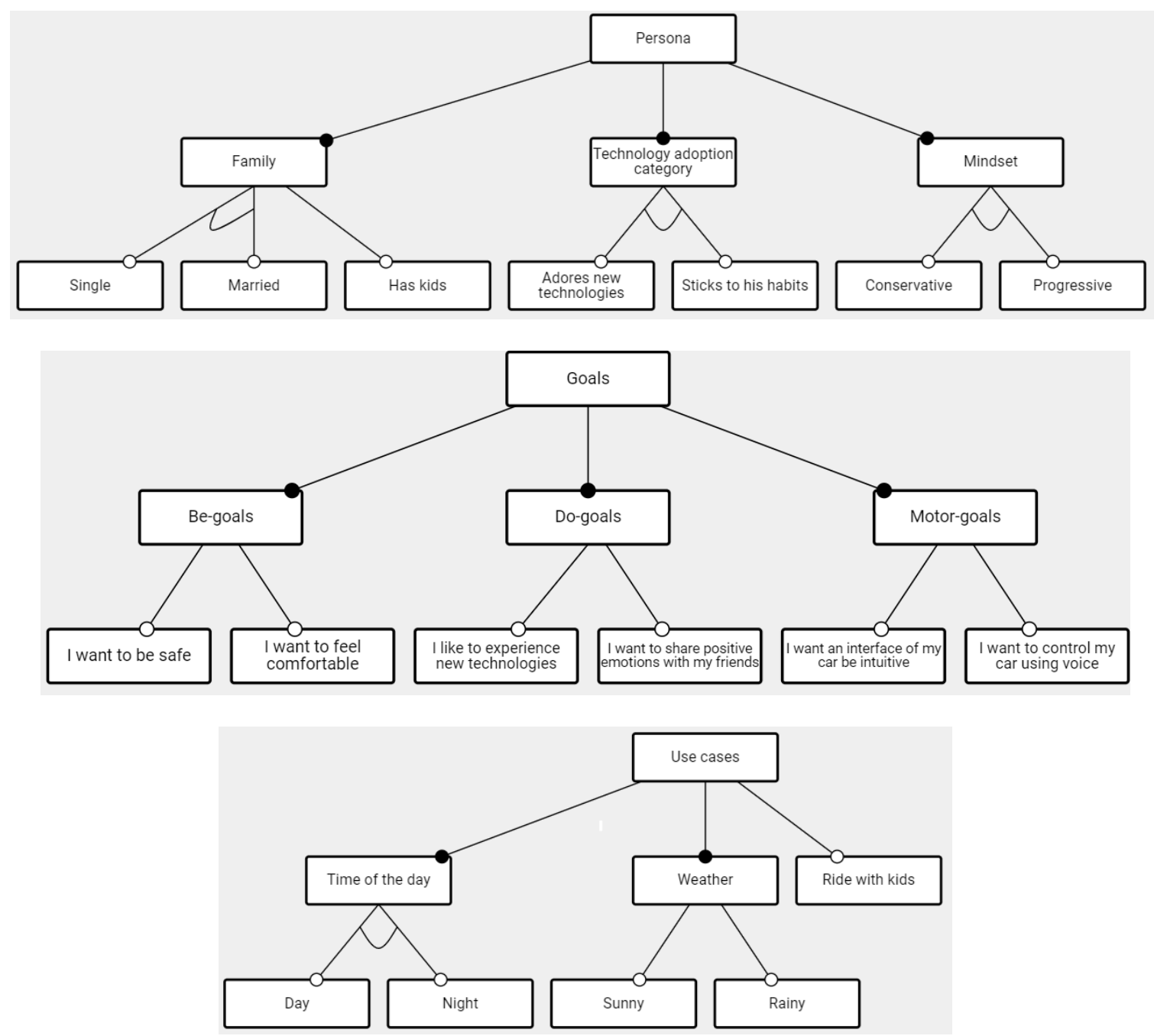

Figure 3. UX concepts representation with feature trees

The setting for the sample roadmap is the following. Three global UX trends influence our design, namely the need to integrate car interfaces with mobile phones, mass-customization, and computeraided safety. Particular product versions are designed in the view of three personas - Alice (enthusiast for new technologies; likes bold aesthetics), Bob (conservative both aesthetically and toward innovations), and David (appreciates innovations, conservative aesthetically). Products are designed taking into account concrete user goals and use cases. According to Kim et al. (2018), having these 
elements explicitly represented in the roadmap promotes better coordination between a design team with product and engineering teams.

Particular products are shown in the roadmap following the order of their introduction to a market. Key technologies are located at the lowest level of the roadmap, enabling these products.

The models from the scoping space represented in figure 3 are mapped to the elements of a planning space represented in Figure 4 through FODA's basic configuration mechanism. Figure 5 illustrates this concept for a particular persona. User goals, use cases, and product features are connected with the same syntax and follow the same pattern. In certain contexts, some elements exist almost permanently (e.g., personas, user goals). In such a case, start and end dates may have no sense, in contrast to transient concepts (e.g., product configurations, UX trends). In some other contexts, personas, user goals, and use cases are also transient and represent openings and closures of market opportunities.

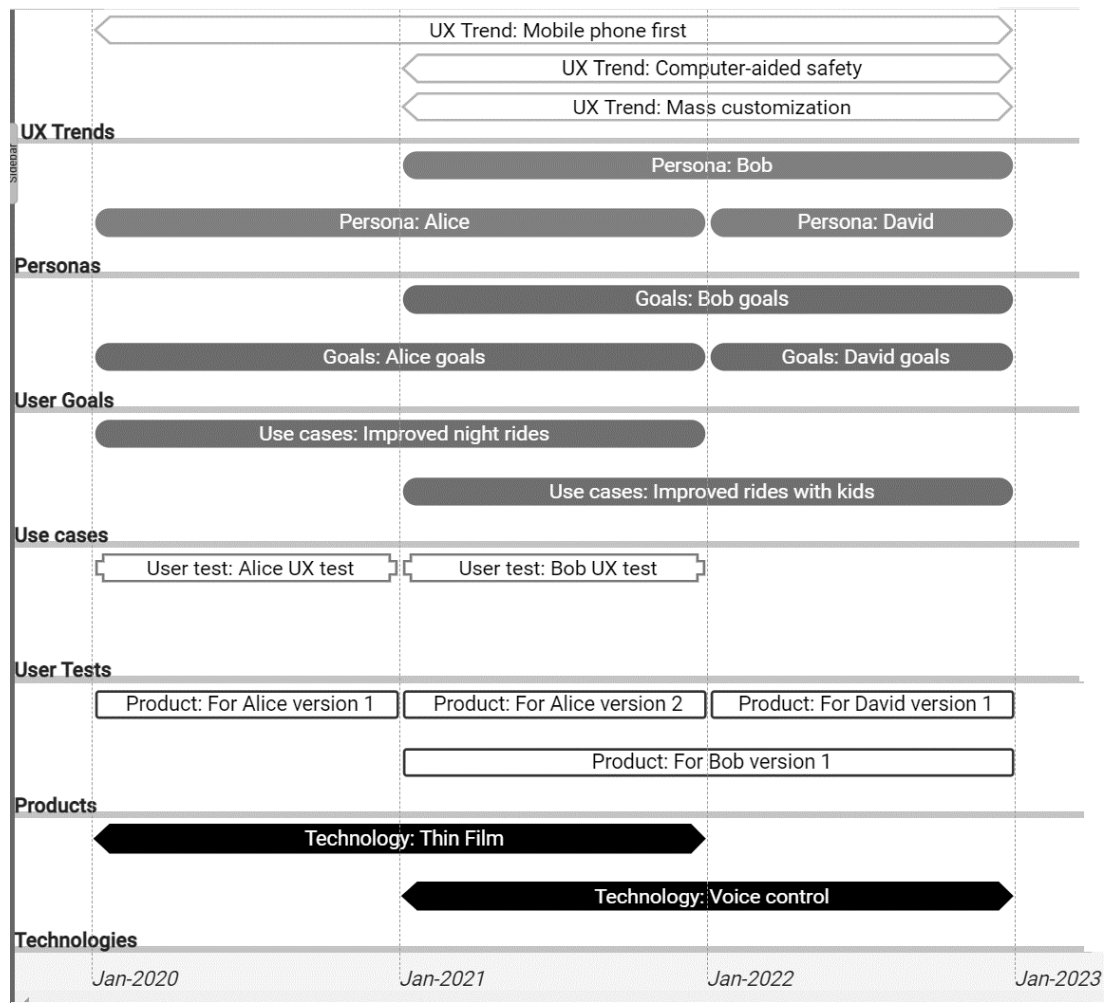

Figure 4. Design roadmap for a novel human-machine interface for semi-automated vehicle

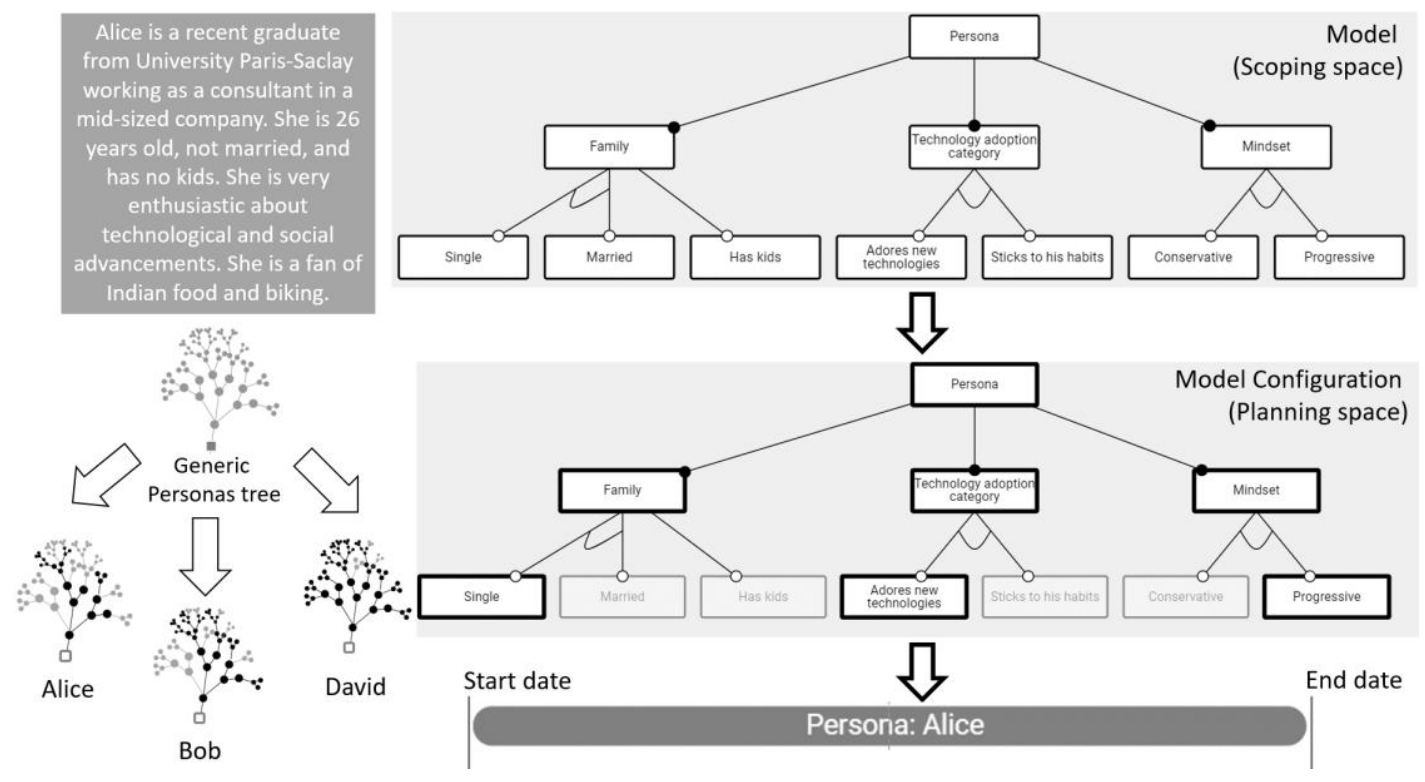

Figure 5. Example of feature tree instantiation 
As was already mentioned, one of the biggest advantages of the proposed approach is the tight integration with user testing. The proposed process is described in the following. In the beginning of the test, a participant fills the KE questionnaire to indicate his/her emotional/aesthetical profile (see fig. 6). Then, another KE questionnaire with different sets of adjectives is filled to assess the product's perceived aesthetics. Then, CA session is conducted (it is shown in figure 7). Following the concept of FODA-CA mapping, options were generated automatically from the feature tree that represents all possible product versions. The final screen is for the point allocation method applied to user stories (see fig. 8). The format of a user story is the following: "I want <feature> in order to <goal> when/during <use case>". The expressions used in user stories are linked to concepts of the corresponding feature trees.

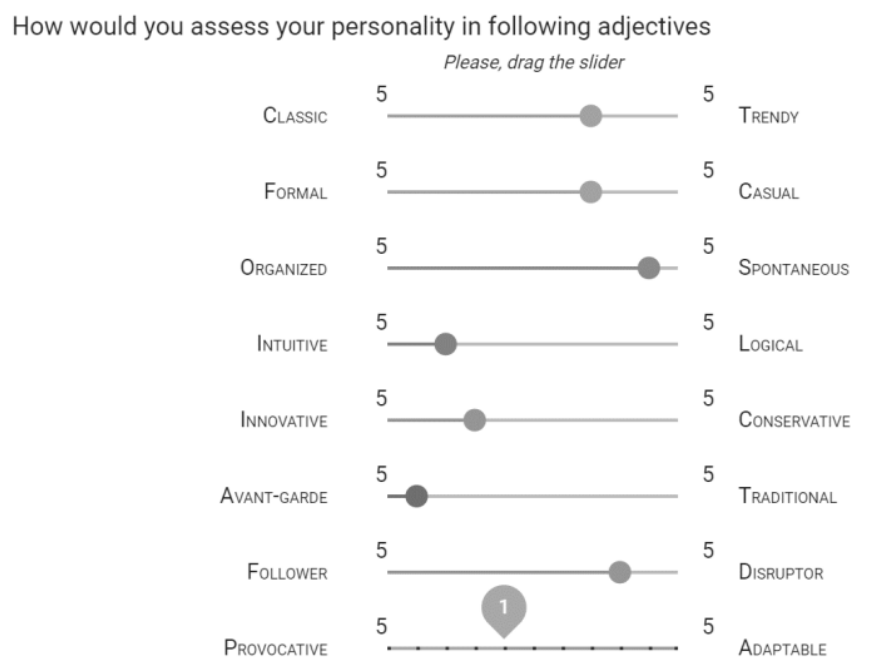

Figure 6. Kansei words self-assessment page

Imagine yourself in a car showroom in 6 month from now buying a new car. Please, rate proposed options (from 0-10). The
price of each option will be added to a basic price of a car. Do not hesitate to downrate options if you consider it overpriced.

Highlighted features indicate the differences between product options, i.e. features that are not highlighted are common for all of the proposed product variants.

\section{Option 1}

Augumented reality windshield projection

Haptic steering wheel

Manual mode

Autonomous mode (L3)

Localized vocal signals

Ambient lighting

Price: 6500 EUR

Please, drag the slider to rate this option

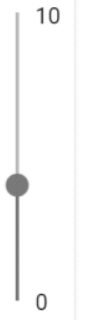

10

\begin{tabular}{l|l} 
Option 2 & \\
Augumented reality windshield projection & \\
Voice control & \\
Haptic steering wheel & \\
Manual mode & \\
Autonomous mode (L3) & \\
Ambient lighting & \\
Price: 6400 EUR & \\
Please, drag the slider to rate this option &
\end{tabular}

$<$ Go back

Figure 7. Conjoint analysis test page

In reality, user test participants first went through a lengthy 3-hour procedure consisting of testing the cockpit prototype in our laboratory, collecting the real-time physiological measurements, going through series of interviews and questionnaires. The test described here was considered a side project; it was conducted online after the main session. Users might choose not to participate in this additional session. At the moment of paper submission, we received answers from 11 participants. The results after data processing are shown in Figure 9. We divided results into two groups based on KE selfassessment using k-means clustering: progressive (closer to Alice) and conservative (closer to Bob and 
David). The responses collected in the following stages were analyzed for these two groups separately. Despite a small sample, the results of this test correspond to the conclusions made based on the interviews.

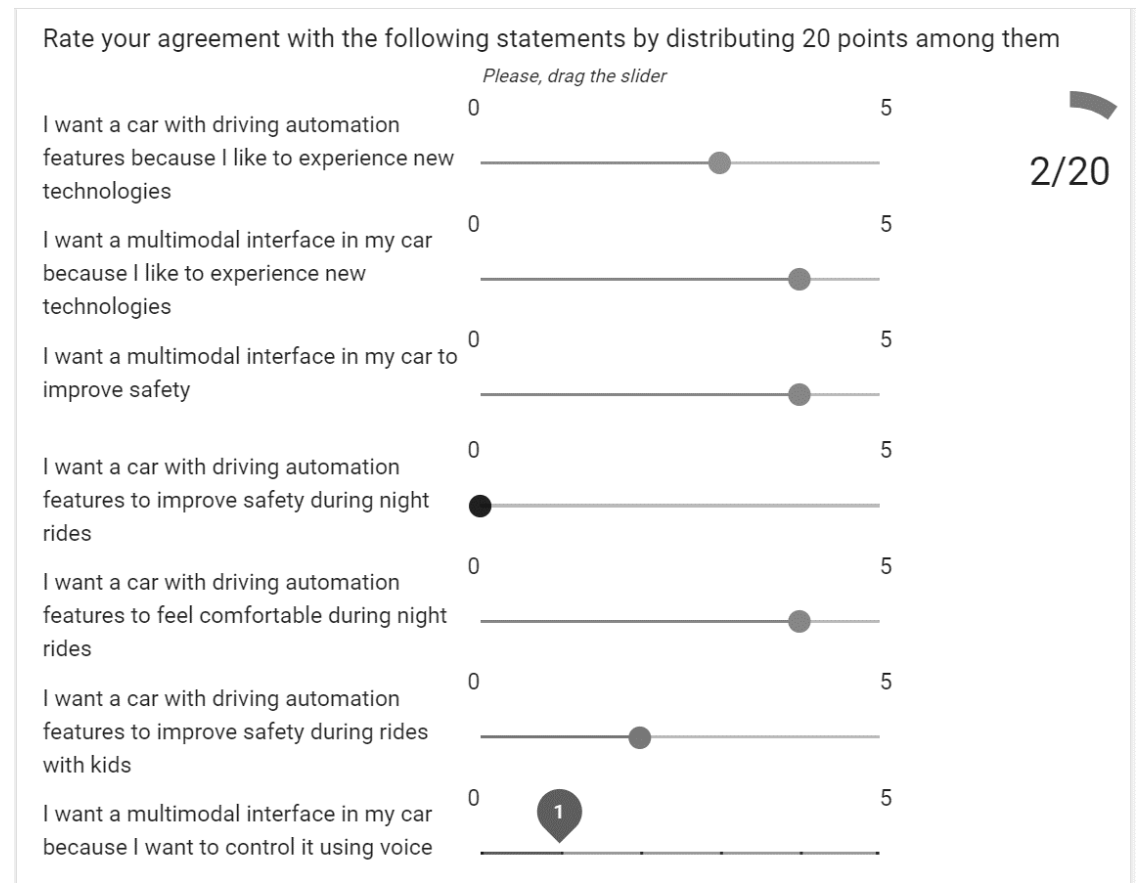

Figure 8. User stories assessment page

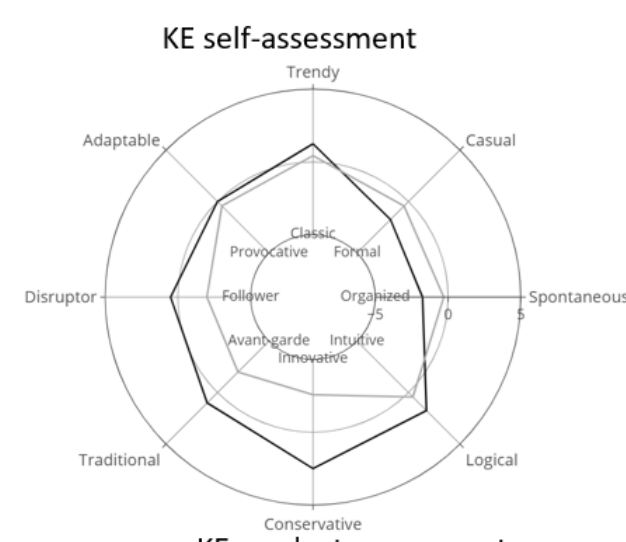

KE product assessment

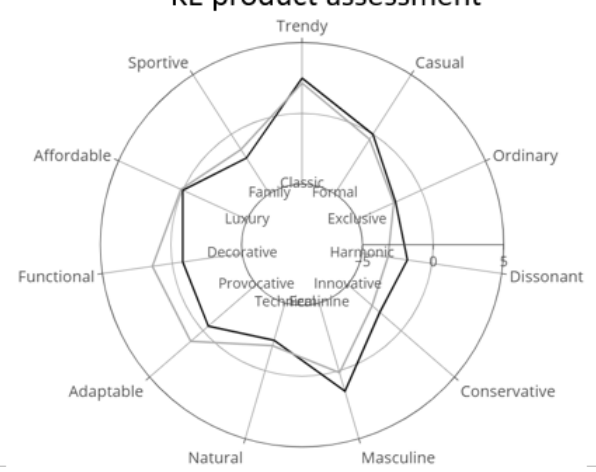

Figure 9. User testing results processing

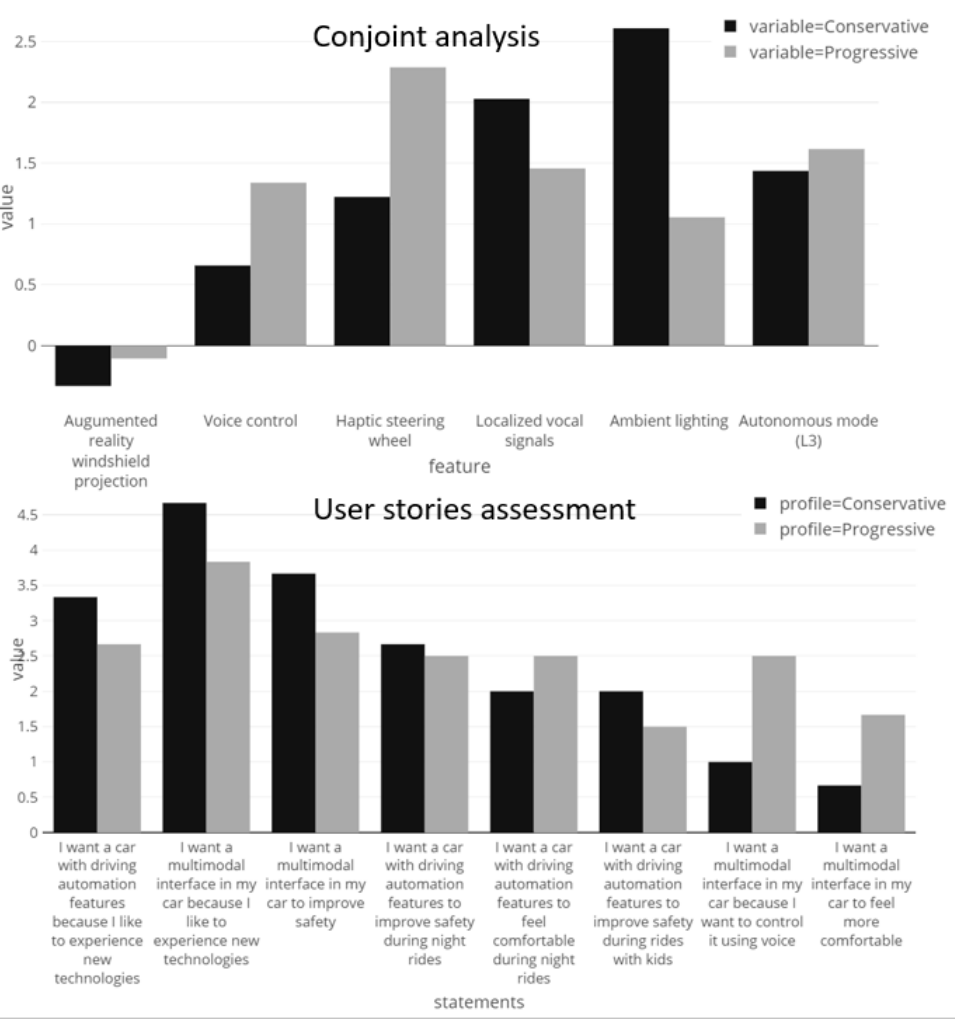

\section{CONCLUSIONS}

In this paper, we suggested that MB-TRM enables the integration of various NPD tools and proposed a concrete approach to achieve such compatibility. The resulted framework expands the scope of MBTRM to UX-focused design roadmapping. 
The authors tailored MB-TRM to the automotive industry context by instantiating general MB-TRM metamodel. Then, we implemented and deployed a web-based software application combining design roadmapping, FODA, and various user testing approaches. The software functionality is demonstrated on the example of a novel human-machine interface design. Finally, we compared the user testing results with the interviewing. The conclusion is that the proposed routine could be considered a fast and affordable solution for hypothesis testing during customer development, feature prioritization, and product family planning.

The overall validation of the methodology as a novel NPD instrument is left for future work. Nevertheless, some preliminary conclusions could be drawn from the discussions of our approach with several practitioners from the automotive and IT fields. First, adopting such a tool will enforce standardization of the UX-related concepts among different departments of a company (namely, design, product, engineering, marketing, and executive managers), reducing conflicts and improving communication efficiency. Second, formal syntax offers a number of opportunities for consistency checks and work automation. The capability of storing the standardized concepts in computer memory promotes information reuse (e.g., personas can be reused between several projects). Third, the roadmap's hierarchical structure helps to keep separation of concerns, permitting for some to see the global picture and for others - more detailed information belonging to the domain of interest, while the consistency check happens behind the scene. Fourth, integration with user tests helps to organize an inexpensive iterative data-driven process of product planning.

In future works, we plan to incorporate UX conceptual models into our approach and test it against other UX elaboration tools (e.g., user journeys) and validate our preliminary conclusions more rigorously.

\section{ACKNOWLEDGEMENTS}

The authors would like to thank Virgil Rousseaux for his contribution to data gathering and for the insightful discussions.

\section{REFERENCES}

“2015 NASA Technology Roadmaps (Archive)”. (2015), NASA, Text, 29 April, available at: http://www.nasa.gov/offices/oct/home/roadmaps/index.html (accessed 24 July 2020).

Abrantes, R. and Figueiredo, J. (2014), "Feature based process framework to manage scope in dynamic NPD portfolios", International Journal of Project Management, Vol. 32 No. 5, pp. 874-884. http://doi.org/10.1016/j.ijproman.2013.10.014

Alexander, I.F. and Maiden, N. (Eds.). (2004), Scenarios, Stories, Use Cases: Through the Systems Development Life-Cycle, 1st edition., Wiley, Chichester; Hoboken, NJ.

An, Y., Lee, S. and Park, Y. (2008), "Development of an integrated product-service roadmap with QFD: A case study on mobile communications", International Journal of Service Industry Management, Emerald Group Publishing Limited, Vol. 19 No. 5, pp. 621-638. http://doi.org/10.1108/09564230810903497

Bekhradi, A., Yannou, B., Cluzel, F. and Kokkolaras, M. (2018), "Decision Support for R\&D Activities of Innovative Technologies", presented at the ASME 2018 International Design Engineering Technical Conferences and Computers and Information in Engineering Conference, American Society of Mechanical Engineers Digital Collection. http://doi.org/10.1115/DETC2018-85657

Buchanan, R. (1992), "Wicked Problems in Design Thinking”, Design Issues, Vol. 8 No. 2, pp. 5-21.

Colombo, E.F., Shougarian, N., Sinha, K., Cascini, G. and de Weck, O.L. (2020), "Value analysis for customizable modular product platforms: theory and case study", Research in Engineering Design, Vol. 31 No. 1, pp. 123-140. http://doi.org/10.1007/s00163-019-00326-4

Doyle, J.R., Green, R.H. and Bottomley, P.A. (1997), “Judging Relative Importance: Direct Rating and Point Allocation Are Not Equivalent”, Organizational Behavior and Human Decision Processes, Vol. 70 No. 1, pp. 65-72. http://doi.org/10.1006/obhd.1997.2694

Du, X., Jiao, J. and Tseng, M.M. (2016), "Architecture of Product Family: Fundamentals and Methodology":, Concurrent Engineering, Sage Publications Sage CA: Thousand Oaks, CA. http://doi.org/10.1177/1063293X0100900407

Franceschini, F. and Rossetto, S. (1995), “QFD: The problem of comparing technical/engineering design requirements", Research in Engineering Design, Vol. 7 No. 4, pp. 270-278. http://doi.org/10.1007/BF01580463

Golkar, A. and Garzaniti, N. (2020), "Model based systems engineering approach to technology roadmapping", Proceedings of the 2020 Summer Simulation Conference, Society for Computer Simulation International, San Diego, CA, USA, pp. 1-12 
Gradini, R., Salgueiro, S., Budideti, J. and Golkar, A. (2019), "Model-based technology roadmapping: potential and challenges ahead", Proceedings of the 2019 Summer Simulation Conference, Society for Computer Simulation International, Berlin, Germany, pp. 1-11. http://doi.org/10.5555/3374138.3374160

Green, P.E., Krieger, A.M. and Wind, Y. (2001), “Thirty Years of Conjoint Analysis: Reflections and Prospects", Interfaces, Vol. 31 No. 3-Supplement, pp. 56-73. http://doi.org/10.1287/inte.31.4.56.9676

Hassenzahl, M. (2018), "The Thing and I: Understanding the Relationship Between User and Product”, in Blythe, M. and Monk, A. (Eds.), Funology 2: From Usability to Enjoyment, Springer International Publishing, Cham, pp. 301-313. http://doi.org/10.1007/978-3-319-68213-6_19

Helms, B. and Shea, K. (2012), "Computational Synthesis of Product Architectures Based on Object-Oriented Graph Grammars”, Journal of Mechanical Design, American Society of Mechanical Engineers Digital Collection, Vol. 134 No. 2. http://doi.org/10.1115/1.4005592

Iuskevich, I., Hein, A., Amokrane-Ferka, K., Doufene, A. and Jankovic, M. (2021), "A metamodel of an informational structure for model-based technology roadmapping", [Manuscript Submitted for Publication].

Kett, S.G., Schmitt, B. and Wartzack, S. (2017), "What the Statistics Tell Us-How to Use Empiric Data in Design for Emotional Impressions", in Chakrabarti, A. and Chakrabarti, D. (Eds.), Research into Design for Communities, Volume 2, Springer, Singapore, pp. 659-669. http://doi.org/10.1007/978-981-10-3521-0_56

Kim, E., Beckman, S.L. and Agogino, A. (2018), "Design Roadmapping in an Uncertain World: Implementing a Customer-Experience-Focused Strategy":, California Management Review, SAGE Publications Sage CA: Los Angeles, CA. http://doi.org/10.1177/0008125618796489

Kim, E., Chung, J., Beckman, S. and Agogino, A.M. (2016), "Design Roadmapping: A Framework and Case Study on Planning Development of High-Tech Products in Silicon Valley", Journal of Mechanical Design, American Society of Mechanical Engineers Digital Collection, Vol. 138 No. 10. http://doi.org/10.1115/1.4034221

Kim, H., Chen, J., Kim, E. and Agogino, A.M. (2017), "Scenario-Based Conjoint Analysis: Measuring Preferences for User Experiences in Early Stage Design”, presented at the ASME 2017 International Design Engineering Technical Conferences and Computers and Information in Engineering Conference, American Society of Mechanical Engineers Digital Collection. http://doi.org/10.1115/DETC2017-67690

Knoll, D., Golkar, A. and de Weck, O. (2018), “A concurrent design approach for model-based technology roadmapping”, 2018 Annual IEEE International Systems Conference (SysCon), presented at the 2018 Annual IEEE International Systems Conference (SysCon), pp. 1-6. http://doi.org/10.1109/SYSCON.2018.8369527

Meinel, M., Eismann, T.T., Baccarella, C.V., Fixson, S.K. and Voigt, K.-I. (2020), "Does applying design thinking result in better new product concepts than a traditional innovation approach? An experimental comparison study", European Management Journal. http://doi.org/10.1016/j.emj.2020.02.002

Muller, J. and Lillack, M. (2011), "Conjoint Analysis of Software Product Lines: A Feature Based Approach", 2011 37th EUROMICRO Conference on Software Engineering and Advanced Applications, presented at the 2011 37th EUROMICRO Conference on Software Engineering and Advanced Applications, pp. 374377. http://doi.org/10.1109/SEAA.2011.73

Nagamachi, M. (2002), "Kansei engineering as a powerful consumer-oriented technology for product development", Applied Ergonomics, Vol. 33 No. 3, pp. 289-294. http://doi.org/10.1016/S00036870(02)00019-4

Nešić, D., Krüger, J., Stănciulescu, Ștefan and Berger, T. (2019), "Principles of feature modeling”, Proceedings of the 2019 27th ACM Joint Meeting on European Software Engineering Conference and Symposium on the Foundations of Software Engineering, Association for Computing Machinery, New York, NY, USA, pp. 62-73. http://doi.org/10.1145/3338906.3338974

Phaal, R., Farrukh, C.J.P. and Probert, D.R. (2005), “Developing a technology roadmapping system”, presented at the A Unifying Discipline for Melting the Boundaries Technology Management:, pp. 99-111. http://doi.org/10.1109/PICMET.2005.1509680

Ries, E. (2011), The Lean Startup: How Constant Innovation Creates Radically Successful Businesses, Trade Paperback edition., Portfolio Penguin, London.

Shen, X.X., Tan, K.C. and Xie, M. (2000), “An integrated approach to innovative product development using Kano's model and QFD”, European Journal of Innovation Management, Vol. 3 No. 2, pp. 91-99. http://doi.org/10.1108/14601060010298435

Wu, D., Zhang, L.L., Jiao, R.J. and Lu, R.F. (2013), "SysML-based design chain information modeling for variety management in production reconfiguration”, Journal of Intelligent Manufacturing, Vol. 24 No. 3, pp. 575-596. http://doi.org/10.1007/s10845-011-0585-6

Zhou, F., Jiao, J.R., Yang, X.J. and Lei, B. (2017), “Augmenting feature model through customer preference mining by hybrid sentiment analysis", Expert Systems with Applications, Vol. 89, pp. 306-317. http://doi.org/10.1016/j.eswa.2017.07.021 